\title{
ZFAS
}

\section{Hebel statt Alibi. Israel und (die) neue deutsche Außenpolitik}

\author{
Moshe Zimmermann
}

(C) Die Autor(en) 2014. Dieser Artikel ist auf Springerlink.com mit Open Access verfügbar.

Zusammenfassung Im Rahmen einer neuen deutschen Außenpolitik müsste mit Blick auf Israel die Gegenwart der Vergangenheit neu bedacht und der historische Zusammenhang zwischen dem Staat Israel und der Shoah in neuer Form in die Einstellung zum Nahen Osten eingeordnet werden. Die „besondere Verantwortung“ Deutschlands Israel gegenüber wird aufgrund einer neuen Einstellung zur europäischen Vergangenheit zum Ausdruck kommen. Dabei wird Deutschland im Rahmen der EU eine aktivere und konstruktive Nahostpolitik betreiben können.

Schlüsselwörter Außenpolitik · Israel · Deutschland · Shoah · Verantwortung

\section{No Alibi Any More. Israel and The New German Foreign Policy}

\begin{abstract}
Israel's relations with Germany are influenced primarily by the presence of the past. Most prominent is the memory of the Shoah. Within the framework of a new German foreign policy towards Israel the nature of this relationship between past and present, between the Shoah and the existence of Israel should be reassessed. Germany's "special responsibility" for Israel will find a new expression thanks to a new interpretation of Europe's past, so Germany can play a more active and constructive role within the framework of European Near-East policy.
\end{abstract}

Keywords Foreign policy · Israel · Germany · Shoah · Responsibility

M. Zimmermann $(\bowtie)$

The Richard Koebner Minerva Center for German History,

The Hebrew University of Jerusalem,

Mount Scopus,

91905 Jerusalem, Israel

E-Mail: moshe.zimmermann@mail.huji.ac.il 
Auf Anregung des deutschen Bundespräsidenten, des deutschen Außenministers und der deutschen Verteidigungsministerin kam es jüngst zu einer umfassenden Diskussion über eine neue deutsche Außenpolitik. Ein Bedarf für eine derartige Neuausrichtung, mindestens aber der Wunsch nach einer neuen deutschen Außenpolitik - diese ist im folgenden Beitrag stets mit der bundesrepublikanischen gleichzusetzen - ergab sich in der Vergangenheit schon mehrmals, im Durchschnitt etwa alle zwanzig Jahre: Zwischen 1949 und 1951 musste die damals noch sehr junge "alte" Bundesrepublik ihre Außenpolitik überhaupt erst einmal festlegen und formen. Zwanzig Jahre später, also etwa ab 1970, kam es zu einer ersten großen Revision und akzentuellen Wende - es wurde die in den breiteren Kontext der Entspannungspolitik eingebettete Ostpolitik entwickelt. Wieder vergingen dann zwei Jahrzehnte, bis das mit dem sogenannten „Ende der Geschichte“ (Francis Fukuyama) zusammenfallende Ende des Kalten Krieges eine weitere Neuorientierung deutscher Außenpolitik notwendig machte. Ab 1990 musste dann eine Außenpolitik für das wiedervereinigte Land gefunden und betrieben werden. Fortan handelte es sich nicht mehr um die Außenpolitik eines Grenzlandes des westlichen Bündnissystems, sondern um die Politik eines Staates, der gewissermaßen in die Mitte Europas gerückt war und nun von einer Pufferzone europäischer Nachbarn und Verbündeter umgeben war. Weitere zwanzig Jahre vergingen, in denen zunehmend die Globalisierung zum Slogan der auswärtigen Politik wurde. In jenen Jahren boten sich genügend Anregungen, über eine ,neue Außenpolitik“ nachzudenken. Sowohl die europäische als auch die globale Dimension deutscher Außenpolitik erlaubten keinen Stillstand. Insgesamt darf es also nicht verwundern, dass stets mehr als nur die real existierende Außenpolitik zur Debatte stand, und die theoretische Diskussion um die grundlegenden Prinzipien einer deutschen Außenpolitik gleichfalls in Bewegung geriet. Eine vierte Periode ist gegenwärtig nicht nur erforderlich, sondern zeichnet sich bereits am Horizont ab.

\section{Zur Geschichte der deutsch-israelischen Beziehungen}

Was sich schematisch so knapp und prägnant zusammenfassen lässt, scheint aus einer spezifischen Perspektive allerdings komplexer, verwirrender und widersprüchlicher zu sein. Im folgenden Beitrag soll es um Israel im Rahmen der deutschen Außenpolitik gehen, für das die skizzierten allgemeinen Schwer- und Wendepunkte nicht unbedingt immer absolut im Einklang stehen mit der ganz eigenen Entwicklungsdynamik und ihren spezifischen Tendenzen. Dies wird ganz deutlich, wenn man sich fragt, welche außenpolitischen Wendepunkte bisher die Beziehung Deutschlands zum Staate Israel und zum Nahen Osten überhaupt markiert haben? Zwei Daten kommen in diesem Zusammenhang automatisch in den Sinn - 1951/52 und 1965. In den Anfangsjahren der Bundesrepublik fügte sich auch die Einstellung zu Israel in den größeren außenpolitischen Rahmen ein: Als sich die Politik Westdeutschlands in der zweigeteilten Welt und im Rahmen des Kalten Krieges positionierte, führte die deutsche Außenpolitik unmittelbar zum Wiedergutmachungsabkommen von 1952, bei dem der 1948 gegründete Staat Israel Partner des Abkommens war.

Auch die zweite Periode deutscher Außenpolitik weist auf eine Schnittmenge oder Deckung zwischen dem weiteren und dem spezifischen Rahmen hin: Es gab durch- 
aus einen Zusammenhang zwischen der bundesrepublikanischen Ostpolitik bzw. der DDR-Politik und der deutschen Israelpolitik, selbst wenn es in diesem Kontext zu einem Durchbruch, einer Wende, bereits während der ersten Periode deutscher Außenpolitik kam, also bevor Bundeskanzler Willy Brandt eine neue Ostpolitik einleitete. Hier ist die bereits 1965 erfolgte Aufnahme offizieller diplomatischer Beziehungen zwischen der Bundesrepublik und dem Staat Israel der Markstein. Die zweite Periode deutscher Außenpolitik bis zur Vereinigung beider deutscher Staaten dauerte deswegen aus israelischer Perspektive länger als die Zeit der allgemeinen neuen Ostpolitik. Sie war zudem eher durch eine Abwesenheit von Konstanz und Stabilität gekennzeichnet: Die Schmidt-Begin-Krise der Jahre 1980/81, bei der es vordergründig um militärische Hilfen aus Deutschland für Saudi-Arabien ging, schien sogar eine Kehrtwende einzuleiten, bis Helmut Kohl, ein Mann mit der „Gnade der späten Geburt", mit und nach seinem Besuch in Israel 1984 bereits vorhandene oder drohende Risse kitten konnte.

Die dritte Periode deutscher Außenpolitik, nämlich die des wiedervereinigten Deutschlands, brachte im Hinblick auf Israel keine eigentlich neue Dimension ins Spiel. Vor dem Hintergrund der ersten Reaktion des damaligen israelischen Ministerpräsidenten Yitzhak Shamir auf den Fall der Berliner Mauer 1989, er wittere nun eine neue Gefahr für Juden, war das jedoch keineswegs selbstverständlich. Eine ablehnende Haltung Israels gegenüber dem territorial und demographisch gewachsenen bundesrepublikanischen Deutschland - man sprach in Israel bisweilen auch vom „Vierten Reich“ - hätte durchaus zu einer reservierten Reaktion der deutschen Seite führen können. Doch haben sich die israelischen Außenminister in der Zeit der deutschen Wiedervereinigung, Moshe Arens und David Levy, ebenso wie der damalige Vize-Außenminister Benjamin Netanyahu für eine Fortsetzung der guten Beziehungen zur Bundesrepublik eingesetzt, während in Deutschland auch Bundeskanzler Helmut Kohl seine Haltung gegenüber Israel in den Jahren bis zum Regierungswechsel 1998 nicht änderte. Daher gab es in den deutsch-israelischen Beziehungen keinerlei Anlass, nach dem Fall der Mauer eine neue Richtung einzuschlagen.

Während des Golfkriegs von 1991 hatte sich der damalige deutsche Außenminister Hans-Dietrich Genscher sehr um eine Unterstützung Israels bemüht, das den Angriffen irakischer Raketen maßgeblich ausgesetzt war. Genscher wurde im Gegenzug für seine Scheckbuchpolitik in den israelischen Medien heftig kritisiert. Grundlegend geändert hat sich in den bilateralen Beziehungen dadurch jedoch nichts. In der Zeit der Rot-Grünen-Koalition - und der zweiten palästinensischen Intifada - sorgte vor allem Außenminister Joschka Fischer für gute Beziehungen zwischen beiden Ländern; sogar als Vermittler zwischen Palästinensern und Israelis konnte er aufgrund des ihm entgegengebrachten Vertrauens aktiv werden. Seit Angela Merkel nun die dritte Bundesregierung anführt, ist Israels Existenz gar zur deutschen Staatsräson aufgewertet worden.

Die Schlüsselworte, die bisher in unseren Ausführungen die kritischen Punkte kennzeichneten, lauten also Kalter Krieg, Ostpolitik, Wiedervereinigung, Europa und Globalisierung. Die Beziehungen zu Israel werden durch diese Markierungen allerdings nur peripher tangiert. Sie stehen vornehmlich mit einem ganz anderen, nicht weniger zentralen Begriff in Verbindung, nämlich dem der Vergangenheitsbewältigung. Eigentlich sollte man besser von dem Konzept einer Gegenwart der Ver- 
gangenheit sprechen. Zwar ist die sogenannte Vergangenheitsbewältigung auch eng mit den oben angeführten Schlüsselbegriffen und ihren konzeptuellen Ideen - Teilung Deutschlands, Ostgrenze, Wiedervereinigung und europäische Idee - verknüpft. Aber in den Beziehungen zu Israel dominiert in erster Linie die Erinnerung an den besonderen Abschnitt der Vergangenheit, den man allgemein Holocaust oder - in Israel - Shoah nennt, also die Erinnerung an die Verfolgung und Ermordung der deutschen und europäischen Juden zwischen 1933 und 1945. Dass die Schatten dieser Vergangenheit des Dritten Reiches auch in anderen von der Bundesrepublik gepflegten bilateralen Beziehungen - wie etwa zu Holland, Frankreich, Griechenland oder Polen, um nur einige relevante Beispiele zu nennen - eine lange Nachwirkung haben, ist eine Binsenweisheit. Aber dieser eine Aspekt ist in den Beziehungen Deutschlands zu keinem anderen Staat so zentral, nachhaltig und wirkungsmächtig, wie in den Beziehungen zu Israel und somit - direkt oder indirekt - zum Nahen Osten.

Auf der Startseite der Homepage der deutschen Botschaft in Tel Aviv wird zusammenfassend betont: „Deutschland steht in einem einzigartigen Verhältnis zu Israel. Aus der Verantwortung Deutschlands für die Shoah, den systematischen Völkermord an den Juden Europas, ist ein dichtes Netz enger Kontakte von Gesellschaft, Wirtschaft, Kultur und Wissenschaft entstanden. Unverkennbar handelt es sich um eine monokausale Formulierung. Wie dieser Grundsatz die Beziehungen zwischen beiden Staaten beeinflussen konnte, zeigt folgendes Beispiel: Stellen wir uns einmal vor, die Olympischen Spiele von 1972 hätten nicht in München, sondern in London oder Bogota stattgefunden. Hätte der Angriff palästinensischer Attentäter auf die olympische Mannschaft Israels beim Gastgeber eine auch nur annähernd vergleichbare Reaktion hervorgerufen, wie sie damals in Deutschland zu verzeichnen war? Und hätte Israel so reagiert, wie es im September 1972 reagiert hat? Die Antwort scheint einfach zu sein: weil das Attentat auf deutschem Boden stattfand, kam nicht nur das Thema des Nahost-Konflikts zwischen Israel und Palästina auf den Tisch, sondern auch die NS-Vergangenheit. Der Verlauf der Ereignisse stand im Zeichen einer spezifischen zwischenstaatlichen Beziehung vor dem Hintergrund einer spezifischen Geschichte und einer besonderen Erinnerungsarbeit.

\section{Zwischen Gegenwart und Vergangenheit}

Dieser Komplex führt uns nun zu der heiklen Frage nach der Bedeutung des jüngst erfolgten Aufrufs zu einer neuen deutschen Außenpolitik im Kontext der deutschen Israel- und Nahostpolitik. Wenn man in deutschen Ministerien eine außenpolitische Revision einleiten und eine neue deutsche Außenpolitik entwickeln möchte, in der Deutschlands internationale Aufgaben neu zu definieren sein werden, so müsste mit Blick auf Israel (und aus dem Blickwinkel von Israel) vor allem auch die Gegenwart der Vergangenheit neu bedacht und die Erinnerung an die Shoah in neuer Form in die Einstellung zum Nahen Osten eingeordnet werden. Denn alle Fragen, die die deutsche Außenpolitik im Allgemeinen beschäftigen - ob es nun um Waffenlieferungen in Kriegsregionen, Entwicklungshilfe oder Wirtschaftsverträge geht - stehen in Bezug auf Israel nolens volens unter dem Zeichen der schwierigen Vergangenheit. Dabei geht es sowohl um die Überlegungen der deutschen Politik als auch um die 
Erwartungen und um die außenpolitische Taktik des Staates Israel. Die israelische Politik lässt nie die Geschichte, vor allem die Geschichte des 2. Weltkriegs und der Shoah, aus dem Blick, und diese Geschichte gilt sodann als Argument - nicht bloß als Hintergrunddekoration.

Zu einem Umdenken im Bereich der Relation zwischen Vergangenheit und Gegenwart im Nahen Osten auch jenseits der spezifischen deutsch-israelischen Beziehungen bzw. des israelisch-palästinensischen Konflikts ist nicht nur die Bundesrepublik Deutschland, sondern wohl die Welt als Ganze aufgerufen, ja praktisch gezwungen: Seit dem Irakkrieg von 2003 werden die alten, seit dem Ende des Ersten Weltkrieges geltenden Staatsgrenzen zunehmend in Frage gestellt oder praktisch verwischt. Mit Beginn des sogenannten ,arabischen Frühlings“ von 2011 sind die politischen Karten in der Region überhaupt völlig neu gemischt. Selbstverständlich ist der ,,arabische Frühling “ für die israelische Außenpolitik eine präzedenzlose Herausforderung, die jedoch am Ende nur die israelische Wagenburg-Mentalität weiter radikalisiert und zur Einigelungspolitik führt. Aus deutscher Perspektive hingegen, deutet sich eine bisher unerwartete historische Entwicklung an; denn die neue Situation, in der sich der Nahe und Mittlere Osten befindet, bedeutet in gewissem Sinne eine Revision des Friedens von Versailles - oder vielmehr des Vertrags von Sevres von 1920 bzw. des späteren Vertrags von Lausanne (1923): Die Grenzen und die Ordnung, die ihren Ursprung im Sykes-Picot Abkommen (1916) hatten, also vom damaligen Lager der Gegner des Kaiserreichs ausgehend, kommen ins Wanken. Zudem ist in dieser Region ein „Krieg der Kulturen“ auf eine äußerst radikale Art erneut ausgebrochen: Das Zeitalter der Religionskriege ist in den Orient zurückgekehrt. Der größere Rahmen für ein Umdenken in dem Beziehungsgeflecht zwischen Israel, Palästina und Deutschland ist für die deutsche Außenpolitik bereits ebenso stringent abgesteckt wie die Bedingungen für einen Paradigmenwechsel im Hinblick auf die Gegenwart der Vergangenheit in der aktuellen Politik.

Hier wie dort muss Geschichte neu studiert und bewertet werden. Die Ausgangsposition für das besagte ,einzigartige Verhältnis“, für die so „,besonderen Beziehungen Deutschlands zu Israel“" war bisher die Hypothese vom kausalen Zusammenhang zwischen der Shoah und der Entstehung des Staates Israel. Diese Hypothese - die in der israelischen Politik weiter unantastbar bleibt - ist nun meines Erachtens als grundsätzlich falsch zu verwerfen. Die Shoah, so hieß es zuerst in der zionistischen Historiographie, dann aber auch in der deutschen Politik, habe zur Gründung des Staates Israel geführt, und zwar entweder, weil sich die zionistische Lösung der Judenfrage angeblich als einzige Alternative zur nationalsozialistischen „Endlösung der Judenfrage“ angeboten, oder einfach, weil die NS-Herrschaft die Flucht von Juden nach Palästina verursacht und begünstigt und so potenziell die Errichtung eines Judenstaates eingeleitet und gefördert habe.

Geht man von dieser irrtümlichen Prämisse aus, so muss jede politische Unterstützung Deutschlands für Israel als eine Sühneleistung des deutschen Staates für die Verbrechen der Shoah gelten. Auf der anderen Seite der Medaille aber zeigt sich: Jede Politik, die nicht die Interessen Israels - und zwar nach dem Verständnis und aus der Sicht der jeweils amtierenden israelischen Regierung - berücksichtigt, gilt als verfehlt. Dies wiederum führt zu einer im Endeffekt automatischen Unterstützung Deutschlands für Israels Politik gegen die arabische Umwelt und vor allem gegen 
die Palästinenser, obwohl Deutschland ausdrücklich theoretisch von einer Politik der „gleichzeitigen Anerkennung“ des „Rechts der Palästinenser auf einen eigenen Staat" ausgeht. Wer in Deutschland oder anderswo aus dieser Sackgasse herauskommen wollte, wandte bisher eine Umgehungstaktik an: Man griff zu einem weiteren Glied im Konstrukt der kausalen historischen Kette und konstruierte die „Wiedergutmachung" wie folgt: Das Elend der Palästinenser resultiere aus der Tatsache, dass sie die Opfer der Opfer des Nationalsozialismus (d. h.: Opfer des Staates, der wegen der Shoah entstand) geworden seien. Aber auch wenn dieses Konstrukt ein Teil der Politik oder mindestens mancher deutscher Denkrichtung geworden ist - ist man von dem ursprünglichen kausalen Grundsatz damit keineswegs abgewichen. Letztendlich beruht die Haltung der deutschen Außenpolitik gegenüber Israel und Palästina bis in die Gegenwart hinein auf der oben skizzierten Ausgangsposition. Die verlängerte kausale Kette aber wird - vielleicht stärker in den Medien als in politischen Proklamationen - bisweilen auch als Rechtfertigung für eine anderenfalls nicht als legitim geltende Kritik an Israel und eine ansonsten offensichtlich nicht begründbare ausgewogene Nahostpolitik nutzbar gemacht.

Nun aber ist die Ausgangsposition mit der Hypothese von einem kausalen Zusammenhang zwischen der Shoah und der Gründung des Staates Israel wie bereits angedeutet grundsätzlich ein Irrtum und daher auch die daraus resultierende Umgehungstaktik ein Irrweg. Der Zionismus - und mit ihm auch die jüdisch-national begründete Auswanderung von Juden nach Palästina - existierte lange vor 1933 und galt als Antwort auf die Assimilation der jüdischen Bevölkerung in ganz Europa, aber auch als Reaktion auf einen gesamteuropäischen Antisemitismus, der den Prozess der Assimilation der Juden begleitete und ablehnte. Das Problem und seine Lösung erweisen sich also als nicht spezifisch deutsch, sondern als allgemein europäisch. Darüber hinaus aber ist zu sagen: Die nationalsozialistische Judenpolitik ging zwar mit der extremsten Art von Antisemitismus einher, gefährdete damit jedoch im Endeffekt erheblich die zionistische Vision vom Judenstaat; denn durch die Ermordung der europäischen Juden reduzierte sie radikal das zionistische Reservoir potentieller jüdischer Auswanderer nach Palästina. Die heute in der „kontrafaktische Geschichte“ nicht mehr für illegitim gehaltene Frage, was aus dem jüdischen Staat ohne den Holocaust geworden wäre, könnte zur Antwort haben: Die Situation im Nahen Osten hätte sich als noch problematischer erwiesen, da die Zahl europäischer Juden in Palästina noch stärker angewachsen wäre. Davon zu trennen ist allerdings die Instrumentalisierung der Erinnerung an die Shoah durch die israelische Politik, die von der Öffentlichkeit unterstützt wird und im öffentlichen Diskurs stets präsent ist, sicher auch im Bewusstsein, mit welcher Grundhypothese zum besagten kausalen Zusammenhang man es in Deutschland und in der westlichen Welt überhaupt zu tun hat.

Es geht hier aber nicht nur um eine rein theoretische Überlegung, die man in der Realpolitik oder auf dem Hintergrund der großen Veränderungen im Nahen Osten marginalisieren oder gar ignorieren könnte. Es handelt sich um eine völlig neue Ausgangsposition, um das Anlegen eines Hebels, mit dem man die Weichen für eine neue außenpolitische Richtung stellen kann. Zum einen wird sich aus einer Revision der Grundhypothese eine neue Gegenwärtigkeit der Vergangenheit ergeben; zum anderen aber wird sich die Relation zwischen einer an der Vergangenheit und einer an der Zukunft orientierten Politik deutlich verschieben. Der deklarative Satz der deut- 
schen Außenpolitik, bedingt „,durch seine Geschichte trägt Deutschland eine besondere Verantwortung für die Sicherheit des Staates Israel“, wird einen neuen Inhalt und eine neue Qualität erhalten: Das Kapitel ,Shoah“ wird auf einer anderen Weise interpretiert und von Deutschland her in Politik umgesetzt. Dies könnte eine Distanzierung von der altbekannten Zurückhaltung, ja bisweilen Untätigkeit, der deutschen Politik gegenüber israelischen Handlungsweisen im Palästinakonflikt signalhaft ermöglichen. Um nun keine Missverständnisse aufkommen zu lassen - die Shoah wird weder geleugnet noch irgendwie untergeordnet oder als zweitrangig eingestuft, wenn der Begriff der „Vergangenheit“ nicht einzig und allein auf dieses Verbrechen bezogen wird. Eher ist es umgekehrt! Das historische Ereignis der Shoah wird aus einer facettenreicheren, mehrdimensionalen Perspektive die Außenpolitik Deutschlands - und auch anderer Staaten - weiterhin beeinflussen und ihre Beziehungen zum Staat Israel neu bestimmen.

Deutschland und Europa trügen eine gemeinsame besondere Verantwortung für Israel, auch wenn die Geschichte ohne den Holocaust verlaufen wäre; denn den Antisemitismus haben nicht die Nationalsozialisten in Deutschland erfunden, und das Scheitern des Experiments der Assimilation und Integration der Juden in Europa hatte sich bereits im Zeitalter der Emanzipation, also im 19. Jahrhundert, abgezeichnet. Dass die ablehnende Haltung in Europa gegen eine gesellschaftliche Integration der jüdischen Bevölkerung zur Gründung einer nationaljüdischen Bewegung, eben zum Zionismus, geführt hat, macht Europa für die Entwicklungen in und um diese Bewegung, und also auch für die Konsequenzen im Zielland der zionistischen Auswanderung, mitverantwortlich. Auch deshalb, weil die Erfindung des modernen Nationalismus in Europa eine immer größer werdende Anzahl von Juden an sich zu einer neuen kollektiven Selbstbestimmung als Nation führte, kann Europa nicht von der Verantwortung für die Folgen im Nahen Osten entlastet werden. Doch die daraus resultierende Präsenz der Vergangenheit in der Gegenwartspolitik unterscheidet sich bei dem neuen Ansatz grundsätzlich von der oben genannten am Holocaust orientierten Auffassung: Erstens geht es um eine gesamteuropäische, nicht allein eine deutsche Verantwortung; zweitens erübrigt oder relativiert sich der taktische Hinweis auf die „Opfer der Opfer“; denn der aus Europa in den Nahen Osten gelangende Nationalismus beeinflusste die jüdische und die arabische Gesellschaft vor Ort gleichermaßen und ganz direkt. Wenn von Palästinensern als Opfern des jüdischen Nationalismus gesprochen wird, kann der Holocaust kein selbständiges Element mehr in der Argumentationskette sein.

Zugegeben - eine besondere, zusätzliche Aufgabe fiele der deutschen Außenpolitik infolge der Katastrophe des Holocaust auch bei dem neuen Denkansatz zu. Doch diese Aufgabe wird sich dann anders als bisher stellen und völlig neu zu formulieren sein. Es wird erstens um die Verantwortung für die Ermordung der Menschen gehen, die sich der nationaljüdischen Bewegung - im Falle eines von einer Katastrophe ungestörten Verlaufs der Geschichte - hätten anschließen können. Es wird um eine kollektive Verantwortung gehen. Die Entschädigung der nach Palästina ausgewanderten überlebenden Personen hingegen findet auf individueller, nicht kollektiver Ebene statt. Die „Entschädigung“ der autochthonen palästinensischen Bevölkerung wird aber dadurch erfolgen, dass das Leiden dieser Bevölkerung eben nicht in eine Verbindung zur Shoah gesetzt wird. Zweitens - und das ist ganz entscheidend - wird 
die Auseinandersetzung mit dem Holocaust zu neuen alternativen Schlussfolgerungen in den Beziehungen zu Israel und zur Region insgesamt führen. Auf diesen Punkt soll später noch ausführlicher eingegangen werden.

\section{Zwischen Deutschland und Europa}

Um eine ausgewogene Nahostpolitik rechtfertigen zu können, bediente sich die Bundesrepublik Deutschland bisher der Politik, oder besser: der Taktik, Europa als „Front" vorzuschieben. Besonders in den Angelegenheiten des Nahen Osten verbarg sich die bundesrepublikanische Außenpolitik gerne hinter der europäischen Fassade. $\mathrm{Da}$ aus israelischer Sicht Ausgewogenheit eher als Synonym für eine pro-arabische bzw. pro-palästinensische Haltung wahrgenommen wird, hat Israel auf derartige Initiativen bislang ablehnend reagiert. Hätte sich die israelische Reaktion nicht auf Europa, sondern nur auf Deutschland gerichtet, hätte der automatische Rückgriff auf die Geschichte der Shoah eine andere Dimension erreicht. Deutsche Politik scheute also aus eben den Gründen, die mit der bisher dominanten Interpretation der Vorgeschichte des Staats Israel zu tun hatten, in ihrer Nahostpolitik den Alleingang. Ob bei der Deklaration der Mitglieder der europäischen Wirtschaftsunion vom 13. Juni 1980 zum arabisch-israelischen Konflikt oder bei der gegenwärtigen Haltung gegenüber Exporten aus israelischen Siedlungen in den besetzten palästinensischen Gebieten - die Bundesrepublik beteuerte immer wieder, dass die Politik in dieser Sache eine gemeinsam europäische sei. Folgt man der Logik des vorliegenden Beitrags, so könnte es zu einem Rollenwechsel in der deutschen Nahostpolitik kommen: Statt sich hinter der Fassade Europas zu verstecken und an die Wünsche anderer europäischer Staaten wie Frankreich oder England anzupassen, könnte Deutschland eine zentrale Rolle übernehmen und Initiative ergreifen. Kurz: Statt Alibi wird Europa zum tragenden Instrument deutscher Nahostpolitik werden. Da in Israel - sowohl von Politikern als auch von den Medien - Deutschland sowieso als wichtigste europäische Macht wahrgenommen wird, könnte diese Wende in Israel mehr auf Zustimmung als auf Widerstand treffen.

Bereits die 1980 erfolgte Deklaration von Venedig zeigte, dass Europa mit Blick auf den Nahen Osten über mehr Erfahrung und vor allem mehr Weitsicht als die Kontrahenten in der Region oder sogar die damaligen Supermächte verfügt. Im Rahmen einer europäischen Politik und mit dem Gewicht der Europäischen Union auf internationalem Boden hat eine europäische Initiative mehr Chancen, konkrete Resultate zu erzielen, als man sich in Europa und Deutschland vielleicht vorzustellen vermag. Wenn nach 1990 nicht nur der Eindruck vom „Ende der Geschichte“, sondern auch von der Allmacht der Vereinigten Staaten entstanden war, so zeichnet sich zweieinhalb Jahrzehnte nach dem Fall der Mauer ein klarer Rückzug der Vereinigten Staaten aus den Angelegenheiten des Nahen Ostens - oder besser: das stark nachlassende Interesse Amerikas an einer Involvierung in der Region - deutlich ab. Dies wiederum öffnet einem größeren Interesse und stärkeren Einfluss der erweiterten Europäischen Union in dieser Region, insbesondere mit Blick auf den israelisch-palästinensischen Konflikt, ein Fenster. Wenn die deutsche Außenpolitik sich von den oben genannten falschen historischen Prämissen zum Zusammenhang zwischen der Shoah und 
dem akuten Konflikt im Nahen Osten befreien kann und stattdessen die europäische historische Dimension des Konflikts in den Vordergrund rückt, dann könnte sie ihre Hemmungen überwinden und eine tragende Rolle bei der Beilegung des Konflikts, also beim Friedensprozess, spielen. Wenn es in Israel zur Überwindung der traditionellen Auslegung der kausalen Beziehung zwischen Shoah und Entstehung des Staates kommen sollte - und das ist die große Schwierigkeit -, wäre die führende Rolle Deutschlands im Rahmen einer neuen europäischen Nahostpolitik aus israelischer Sicht unproblematisch.

Durch einen derartigen Ansatz werden auch die stetigen, quasi automatisch aus Israel kommenden Hinweise auf die deutsche Geschichte zur Zeit des Nationalsozialismus und der Shoah, welche die sachlichen Gegenargumente ersetzen sollen, irrelevant. Der ständige Vergleich mit München 1938, wie er von Menahem Begin nach der Erklärung von Venedig oder jüngst von Benjamin Netanyahu während der Verhandlungen über das nukleare Potenzial des Iran gezogen wurde, aber auch die Erwähnung der „Auschwitz-Grenzen“ und Ähnliches mehr wird auch für die israelische Politik an Wert und Effektivität verlieren; denn die Vergangenheit, die man vergegenwärtigen möchte, wird eine gesamteuropäische Vergangenheit sein und weiter als bis in die erste Hälfte des 20. Jahrhunderts zurückreichen. Wenn Europa für das Entstehen des jüdischen Nationalismus mitverantwortlich zu machen ist, dann kann es und dann kann vor allem auch Deutschland aus der Vergangenheit im Nationalismus schöpfen, um eine Richtlinie für eine Nahostpolitik vorzugeben.

Daraus ergibt sich ein wichtiger neuer Blickpunkt. Europa und damit auch Deutschland kann mit Hilfe seiner Außenpolitik eine auf seiner Erfahrung beruhende postnationale Botschaft in den Nahen Osten bringen. Europa, und wiederum vor allem Deutschland, hat aus der Geschichte des Nationalismus, der seinen Höhepunkt (oder eigentlich Tiefpunkt) in der Zeit des Nationalsozialismus erreicht hatte, vornehmlich gelernt, wie schädlich die Exzesse des nationalistischen Denkens und seiner Politik werden können. Im Namen des aufgeklärten europäischen Nationalismus aber kann eine deutsche Außenpolitik für eine entsprechende Tendenz im Nahen Osten plädieren und darauf hinwirken, dass ähnliche Prozesse im Nahen Osten eingeleitet werden, wie man sie aus Europa seit 1945 kennt. Zugegeben: Die Bereitschaft in Israel auf den militanten Nationalismus zu verzichten, oder nationalistische Parolen aufzugeben ist gering (allerdings auch bei den Palästinensern). Da der Glaube daran, dass die Nation der höchste Wert ist, und dass der Kampf gegen Feinde auch in Zukunft unausweichlich bleibt, gibt es in der israelischen Öffentlichkeit keine Aufnahmebereitschaft für postnationale Botschaften. Doch das typische israelische Argument, „wir sind nicht in der Schweiz“ oder „hier ist nicht Europa, sondern der wilde Osten“, beruht auf einer falschen historischen Vorstellung: Das Europa von heute ist keine prädestinierte Gegebenheit, sondern eine Schlussfolgerung, die man aus einer langen, katastrophalen und blutigen Geschichte gezogen hat. Wenn dies in Europa möglich war, warum sollte es nicht auch im Nahen Osten geschehen können.

Eine neue deutsche Außenpolitik, die im Nahen Osten Positives bewirken möchte, muss darüber hinaus versuchen, allen Kontrahenten in der Region, vor allem Israel, die Vorteile einer föderalen, übernationalen Struktur nach europäischem Modell „schmackhaft“ zu machen. Die Europäische Union als Vorbild den Beteiligten im Nahen Osten zu präsentieren, nicht als Fata Morgana, sondern als reale Alternative, 
wäre eine nützliche Herausforderung für Deutschland im Rahmen der europäischen Außenpolitik. Zugegeben: Solange der Nationalismus, die Angst und vor allem die Behauptung, Israel sei die Antwort, und zwar die einzige, auf die Shoah dominieren, ist der Weg der Vision von einem „neuen Nahen Osten“ holprig. Doch da Europa in der Vergangenheit den alten Nationalismus in diese Region exportieren konnte, wäre auch der Export der postnationalen Botschaft nicht unwahrscheinlich.

\section{Der andere Umgang mit der Shoah in der Außenpolitik}

Und nun gehen wir noch einmal zurück zum Kapitel des Holocaust und seiner Allgegenwart in den deutsch-israelischen Beziehungen: Deutschland in Europa, ein Land, das früher seine Nachbaren für Feinde hielt und heute mit allen ehemaligen Feinden in Frieden lebt, wäre - sicher auch mit allen seinen Schwächen - Lehrstück und Vorbild. Es könnte eine Außenpolitik betreiben, die auf der Auseinandersetzung mit den Erfahrungen im Nationalsozialismus und der Geschichte des Holocaust aufbaut. In unserem Zusammenhang bedeutet dies, anders als im Kontext der oben abgelehnten Prämisse: Die Umsetzung dieser Erfahrung im Rahmen einer deutschen Außenpolitik wird darauf abzielen, Rassismus und Antisemitismus - in Israel und in der Region überhaupt - zu bekämpfen, Demokratie zu verankern sowie den Schutz der Menschenrechte und der Menschenwürde zu garantieren. Hierin fände Deutschland eine optimale, konstruktive Aufgabe seiner Außenpolitik. Daneben wird dann auch der Auftrag treten, die Lehren aus der europäischen Geschichte religiöser Intoleranz zu vermitteln, und die Alternativen der Toleranz und des Laizismus als Gegenentwurf zu einem wohl möglichen, ja beinahe aktuellen Religionskrieg im Nahen Osten aufzuzeigen. Wie bereits erwähnt, wird gegenwärtig der Durchschnittsisraeli wie auch der israelische Durchschnittspolitiker diese Vision mit äußerster Skepsis betrachten - die große Aufgabe des israelischen Erziehungssystem ist es deshalb, die Voraussetzung für diesen Bewusstseinswandel zu schaffen.

Aus der neuen Ausgangsposition ergäbe sich dann zu guter Letzt eine weitere Wahrnehmung mit praktischen Folgen. Unterstützung für Israel wird dann nicht mehr automatisch die Zustimmung zu allem bedeuten, was die jeweilig amtierende israelische Regierung für richtig und angemessen hält. Eine derartige Zustimmung kann dann nicht mehr wirklich effektiv von der Bundesrepublik bzw. von Europa verlangt oder erwartet werden. Wenn Europa der deutschen Außenpolitik nicht als Fassade, sondern als Fundament und Instrument dient - dann erübrigt sich in der deutschen Nahostpolitik der Drang nach Alibi und Versteckspiel. Der Mut, bisweilen Klartext zu sprechen und den Beteiligten die Leviten zu lesen, wird dann nicht mehr als unverbesserlicher Starrsinn, Neo-Kolonialismus oder Antisemitismus gedeutet werden können.

Open Access Dieser Artikel unterliegt den Bedingungen der Creative Commons Attribution License. Dadurch sind die Nutzung, Verteilung, und Reproduktion erlaubt, sofern der/die Originalautor/en und die Quelle angegeben sind. 


\section{Literatur}

Ben-Natan, A., \& Hansen, N. (Hrsg.) (2005). Israel und Deutschland. Dorniger Weg zur Partnerschaft. Köln: Böhlau Verlag.

Brunner, J. (Hrsg.) (2013). Deutsche(s) in Palästina und Israel. Alltag, Kultur, Politik. Tel Aviver Jahrbuch für deutsche Geschichte, Bd. 41. Göttingen: Wallstein Verlag.

Deutsche Botschaft Tel Aviv. Deutsch-Israelische Beziehungen. http://www.tel-aviv.diplo.de/Vertretung/ telaviv/de/04-Politik/Deutschland-Israel/Bilaterale-Beziehungen.html. Zugegriffen: 16. Sept. 2014.

Feldman, L. G. (2012). Germany's foreign policy of reconciliation. from enmity to amity. Plymouth: Rowman \& Littlefield.

Pallade, Y. (2005). Germany and Israel in the 1990s and beyond. Still a 'Special Relationship'?. Frankfurt a. M.: Peter Lang.

Weingardt, M. (2002). Deutsche Israel- und Nahostpolitik. Die Geschichte einer Gratwanderung seit 1949. Frankfurt a. M.: Campus Verlag.

Zimmermann, M. (2002). Deutsche Vergangenheit, Israelisches Gedächtnis. Tel Aviv. (Hebr.). 